\title{
Assessment of serum neopterin as an inflammatory and cardiovascular marker in type 1 and 2 diabetes complicated by diabetic foot syndrome: a comparative study
}

\section{ABSTRACT}

Introduction. Neopterin is a byproduct of nerve transmitter coenzyme that is synthesized and released by macrophages and T-lymphocytes. It is a useful inflammatory marker of diabetes progression, as its levels increase with the progression of the disease from prediabetes to type 2 diabetes (T2D). This study aimed to compare serum neopterin levels between type- 1 and type-2 diabetes patients with diabetic foot syndrome (DFS), and assess the relation between serum neopterin levels and cardiometabolic risk factors.

Materials and methods. This observational cross-sectional study was carried out in the Centre of Diabetes Mellitus in Erbil, Iraq from $1^{\text {st }}$ January to $31^{\text {st }}$ December 2016. A total of 30 healthy subjects and 140 patients with DFS [70 patients with type 1 diabetes (T1D) and 70 patients with T2D] were enrolled in the study. The main outcome measurements included anthropometric measurements, blood pressure, fasting serum glucose, glycated haemoglobin, lipid profile, neopterin and high sensitivity C-reactive protein (hs-CRP).

Results. Serum neopterin levels of T2D patients were significantly $(p<0.001)$ higher than the corresponding levels of T1D patients $(18.6 \pm 2.1 \mathrm{nmol} / \mathrm{L}$ vs. $12.6 \pm$ $1.3 \mathrm{nmol} / \mathrm{L}$ ). The changes in the serum neopterin levels were related to cardiometabolic risk factors. In T1D,

Address for correspondence:

Marwan S Al-Nimer

Hawler Medical University, Erbil, Iraq

e-mail: alnimermarwan@ymail.com

Clinical Diabetology 2018, 7, 2, 91-96

DOI: $10.5603 /$ DK.2018.0002

Received: 25.08.2017

Accepted: 16.01.2018 a significant positive correlation between serum levels of neopterin and diastolic blood pressure were observed, while in T2D the significant positive correlation was found between fasting serum triglyceride levels and neopterin levels. Serum levels of neopterin were insignificantly correlated with hs-CRP in T1D and T2D. Conclusions. In patients with DFS, serum neopterin levels are significantly higher in those with T2D compared with T1D patients. Neopterin levels are not related to the grading of DFS, but are invariably related to cardiometabolic risk factors. (Clin Diabetol 2018; 7, 2: 91-96)

Key words: diabetic foot syndrome, cardiometabolic risk factors, neopterin

\section{Introduction}

World Health Organization (WHO) defined the diabetic foot syndrome (DFS) as ulceration of the foot (distally from the ankle and including the ankle) associated with neuropathy and different grades of ischaemia and infection [1]. Previous studies reported that DFS is a common complication of diabetes and the prevalence up to $27 \%$ of diabetic patients was found [2-4]. Lauterbach et al. analysed the data of 34,198 patients with type 2 diabetes (T2D) and 2,576 patients with type 1 diabetes (T1D) in the United Kingdom and found that DFS prevalence was $7.6 \%$ and $8.5 \%$, respectively [5]. The results of one study showed that in patients with T2D complicated by DFS the inflammatory markers, including high sensitivity $C$-reactive protein, tumour necrosis factor- $\alpha$ (TNF- $\alpha$ ) and interleukin-6, were significantly correlated with cardiometabolic risk factors, 
including body mass index (BMI), serum triglycerides and low density lipoprotein [6]. Poor glycaemic control has been associated with wound infection caused by multidrug resistant microorganisms [7]. Neopterin is a byproduct of nerve transmitter coenzyme that is synthesized and released by macrophages and T-lymphocytes [8]. One study found that neopterin was a useful inflammatory marker in evaluating the progression of diabetes, as its levels increased from prediabetes to T2D [9]. Rao et al. (2010) reported a significant increase in neopterin serum levels in patients with metabolic syndrome $(2.142 \pm 0.038 \mathrm{nmol} / \mathrm{L})$ compared with subjects without metabolic syndrome $(2.020 \pm 0.044 \mathrm{nmol} / \mathrm{L})$ [10]. In gestational diabetes, serum neopterin level is increased, but does not significantly correlate with high-sensitivity C-reactive protein, which is a marker of inflammation [11]. There is evidence suggesting that the serum level of neopterin is a predictor of fatal ischaemic heart disease in T2D [12].

The rationale behind this study was the role of cardiometabolic risk factors in the aetiopathogenesis of the infection/inflammation in DFS, which may explain the differences in serum neopterin levels between patients with different types of diabetes complicated by DFS. This study aimed to assess the serum neopterin levels in patients with T1D and T2D complicated by DFS and to relate these levels to cardio-metabolic risk factors taking into considerations the DFS severity.

\section{Materials and methods}

This cross-sectional observational study was carried out in the Centre of Diabetes Mellitus in Erbil from $1^{\text {st }}$ January to $31^{\text {st }}$ December 2016 . The study was conducted according to the guidelines of the Declaration of Helsinki with approval of the local ethical review board. Informed consent was obtained from each patient before enrolment into the study. The patients were recruited from the Centre of Diabetes Mellitus, and they were allocated randomly using randomization tables. The criteria of inclusion were: type 1 and 2 diabetes (T1D and T2D) complicated by DFS in patients treated with oral hypoglycaemic agents and/or insulin, according to the clinical status of the patient. The diabetic foot syndrome is a clinical condition characterized by the presence of ulceration, signs of inflammation and infection in the presence or absence of gangrene. The Wagner-Meggitt classification was used for grading DFS [13]. We used this classification because it is simple and provides information related to the objective of the study. In addition, our patients were known cases of DFS and some of them had a history of amputation; therefore, the application of other classifications did not offer more advantages.
The Wagner-Meggitt classification system has six grades (0-5) of lesions. The first four grades are based on the physical depth of the lesion and the last two grades are based on the extent of gangrene. The consultant endocrinologist at the diabetes centre performed clinical examination and classified diabetic foot lesions. The $X$-ray as well as specimen culture and sensitivity assessment were done for each patient. The presence of diabetic peripheral neuropathy was determined on the basis of subjective and objective symptoms (pain, numbness, vibration, tactile and temperature sensations, pin prick and ankle reflex) using the scoring of $0=$ absent and $1=$ reduced or present. In those with positive clinical evidence of neuropathy electromyography and nerve conduction studies (including ulnar, sural and common peroneal nerves) were performed to confirm the presence of peripheral neuropathy.

Patients with a history of rheumatic, haematological, neoplastic, renal, liver or thyroid diseases, and patients receiving treatment with anti-inflammatory drugs were excluded from the study.

The patients were divided into 3 groups:

- Group I ( $n=30)$ : Apparently healthy control subjects;

- Group II $(n=70)$ : T1D patients presented with FDS;

- Group III ( $n=70)$ : T2D patients presented with FDS.

Demographic data, medical history and treatment information were collected in the centre. Modifiable risk factors, events or complications, and current therapy were recorded. A person who reported smoking on admission was defined as current smoker. Height, weight, and waist circumference were measured, and body mass index (BMI) was calculated using Quetlete's equation:

body mass index $\left[\mathrm{kg} / \mathrm{m}^{2}\right]=$ weight $[\mathrm{kg}] / \mathrm{height}^{2}[\mathrm{~m}]$

Blood pressure was measured in sitting position and the mean of three readings was taken.

The measurements of blood pressure in the dorsalis pedis and tibialis posterior arteries were not included in the study because a considerable percentage of patients had a history of amputation, which could be a source of bias in the interpretation of the data. Peripheral venous blood was drawn into tubes immediately after admission. Then the samples were centrifuged at $2500 \mathrm{rpm}$ for $10 \mathrm{~min}$ and the sera were separated for determination of fasting serum glucose, $\mathrm{HbA}_{1 \mathrm{c}}(\%)$ and lipid profile. The lipid profile included fasting serum triglycerides and high density lipoprotein-cholesterol. Quantitative determination of serum high-sensitivity C-reactive protein (hs-CRP) (mg/L) and neopterin were carried out using the enzyme-linked immunosorbent assay (ELISA) technique. 
Table 1. Characteristics of the participants of the study

\begin{tabular}{lccc}
\hline Characteristics & Group I $(\mathbf{n}=\mathbf{3 0})$ & Group II $(\mathbf{n}=\mathbf{7 0})$ & Group III (n= 70) \\
\hline Gender (male:female) & $17: 13$ & $54: 16$ & $50: 20$ \\
Age (years) & $52(44-63)$ & $50.5(43-59)$ & $64.5(53-72)$ \\
Residency (urban:rural) & & $36: 34$ & $29: 41$ \\
Duration of diabetes (years) & - & $14(8-23)$ & $9(4-20)$ \\
Duration of diabetic foot syndrome (months) & - & 36 & 24 \\
Previous history of amputation & - & 23 & $35^{*}$ \\
Grading of diabetic foot syndrome & - & 0 & 0 \\
$\quad$ Grade 1 & - & 23 & $13^{* *}$ \\
Grade 2 & - & 25 & 24 \\
Grade 3 & - & 14 & 22 \\
Grade 4 & - & 08 & 11 \\
Grade 5 & & \\
\hline
\end{tabular}

The results are expressed as number and median (range). ${ }^{*} \mathrm{p}=0.04,{ }^{* *} \mathrm{p}=0.05$ compared with Group II. Group I: Healthy subjects, Group II: Type 1 diabetes with diabetic foot syndrome, Group III: Type 2 diabetes with diabetic foot syndrome

Table 2. Results of cardiometabolic risk factors, inflammatory marker and neopterin levels according to the grading of diabetic foot syndrome in Group II patients

\begin{tabular}{|c|c|c|c|c|}
\hline \multirow[t]{2}{*}{ Parameters } & \multicolumn{4}{|c|}{ Grading of diabetic foot syndrome } \\
\hline & $\begin{array}{l}\text { Grade } 2 \\
(n=23)\end{array}$ & $\begin{array}{l}\text { Grade } 3 \\
(n=25)\end{array}$ & $\begin{array}{l}\text { Grade } 4 \\
(n=14)\end{array}$ & $\begin{array}{l}\text { Grade } 5 \\
(n=8)\end{array}$ \\
\hline Body mass index $\left[\mathrm{kg} / \mathrm{m}^{2}\right]$ & $33.5 \pm 2.2$ & $34.9 \pm 3.1$ & $34.4 \pm 2.9$ & $36.2 \pm 3.4$ \\
\hline Waist circumference $[\mathrm{cm}]$ & $85.6 \pm 6.1$ & $87.3 \pm 5.2$ & $88.3 \pm 5.0$ & $90.8 \pm 4.0$ \\
\hline Systolic blood pressure [mm Hg] & $130.6 \pm 3.6$ & $131.9 \pm 3.7$ & $131.0 \pm 3.7$ & $130.1 \pm 2.5$ \\
\hline Diastolic blood pressure [mm $\mathrm{Hg}]$ & $75.1 \pm 3.6$ & $75.8 \pm 3.3$ & $75.3 \pm 3.7$ & $78.1 \pm 2.0$ \\
\hline Fasting serum triglycerides [mg/dL] & $167 \pm 7.8$ & $166.0 \pm 11.2$ & $172.9 \pm 10.9$ & $171.6 \pm 9.1$ \\
\hline High-density lipoprotein cholesterol & $52.9 \pm 3.1$ & $51.9 \pm 4.3$ & $52.1 \pm 3.3$ & $49.9 \pm 3.2$ \\
\hline Fasting serum glucose [mg/dL] & $318.5 \pm 35.8$ & $306.6 \pm 63.4$ & $311.0 \pm 31.4$ & 326. \pm 28.8 \\
\hline Glycated haemoglobin (\%) & $9.6 \pm 1.1$ & $9.5 \pm 1.2$ & $9.0 \pm 1.0$ & $9.0 \pm 0.8$ \\
\hline High-sensitivity C-reactive protein [mg/L] & $5.8 \pm 0.9$ & $5.7 \pm 0.7$ & $5.7 \pm 1.1$ & $5.9 \pm 1.1$ \\
\hline Neopterin [nmol/L] & $12.84 \pm 1.3$ & $12.5 \pm 1.4$ & $12.7 \pm 1.0$ & $12.0 \pm 1.4$ \\
\hline
\end{tabular}

The results are expressed as mean \pm standard deviation (SD). No significant difference between different groups of diabetic foot syndrome grading

\section{Statistical analysis}

Data are expressed as number, percent, and means \pm SD. Unpaired Student's t-test, and differences between percentages were used to evaluate differences between the two groups and a simple (Pearson's) correlation test was used to detect the correlations between the neopterin and other factors related to DFS. For all tests, a two-tailed $p \leq 0.05$ was considered statistically significant. All calculations were made using Excel 2003 program for Windows.

\section{Results}

Table 1 shows that there is an insignificant difference between Group II and Group III patients in demographic characteristics. Twenty-three (32.9\%) patients in Group II had a history of toes or foot amputation compared with thirty-five (50\%) patients in Group III; the difference reached the level of statistical significance $(p=0.04)$. Regarding the grading of DFS at the time of the current study, significantly $(p=0.05)$ higher number of Group II patients had Grade 2 DFS compared with Group III (Tab. 1). Tables 2 and 3 showed no statistically significant difference in cardiometabolic risk factors or hs-CRP levels depending on the grades of DFS in patients with T1D and T2D. Serum levels of hsCRP of Group III patients were significantly $(p=0.000)$ higher than the corresponding levels of Group II patients $(12.5 \pm 2.6 \mathrm{mg} / \mathrm{L}$ vs. $5.7 \pm 0.9 \mathrm{mg} / \mathrm{L})$. Serum levels of neopterin in Group III patients were significantly ( $p=0.000$ ) higher than the corresponding levels in Group II 
Table 3. Cardiometabolic risk factors, inflammatory marker and neopterin levels according to the grading of diabetic foot syndrome in Group III patients

\begin{tabular}{|c|c|c|c|c|}
\hline \multirow[t]{2}{*}{ Parameters } & \multicolumn{4}{|c|}{ Grading of diabetic foot syndrome } \\
\hline & $\begin{array}{l}\text { Grade } 2 \\
(n=13)\end{array}$ & $\begin{array}{l}\text { Grade } 3 \\
(n=24)\end{array}$ & $\begin{array}{l}\text { Grade } 4 \\
(n=22)\end{array}$ & $\begin{array}{l}\text { Grade } 5 \\
(n=11)\end{array}$ \\
\hline Body mass index $\left[\mathrm{kg} / \mathrm{m}^{2}\right]$ & $35.3 \pm 2.3$ & $35.75 \pm 3.26$ & $37.94 \pm 3.7$ & $35.88 \pm 2.98$ \\
\hline Waist circumference $(\mathrm{cm})$ & $94.8 \pm 5.0$ & $97.2 \pm 7.0$ & $98.4 \pm 9.6$ & $96.4 \pm 8.2$ \\
\hline Systolic blood pressure [mm Hg] & $147.2 \pm 7.0$ & $147.3 \pm 4.8$ & $150.6 \pm 3.6$ & $151.2 \pm 3.0$ \\
\hline Diastolic blood pressure [mm Hg] & $89.1 \pm 6.6$ & $89.4 \pm 4.6$ & $91.1 \pm 2.8$ & $90.6 \pm 6.0$ \\
\hline Fasting serum triglycerides [mg/dL] & $198.7 \pm 18.3$ & $196.4 \pm 17.4$ & $196.8 \pm 16.2$ & $207.1 \pm 20.4$ \\
\hline High-density lipoprotein cholesterol [mg/dL] & $45.8 \pm 2.8$ & $46.3 \pm 2.6$ & $45.9 \pm 2.1$ & $46.3 \pm 3.1$ \\
\hline Fasting serum glucose $[\mathrm{mg} / \mathrm{dL}]$ & $223.3 \pm 21.0$ & $225.0 \pm 25.4$ & $220.2 \pm 22.7$ & $223.8 \pm 16.9$ \\
\hline Glycated haemoglobin (\%) & $8.0 \pm 0.96$ & $7.7 \pm 0.7$ & $7.7 \pm 0.6$ & $7.7 \pm 0.8$ \\
\hline High-sensitivity C-reactive protein [mg/L] & $12.0 \pm 1.7$ & $13.17 \pm 2.66$ & $12.48 \pm 3.1$ & $11.6 \pm 2.0$ \\
\hline Neopterin [nmol/L] & $19.04 \pm 2.02$ & $18.11 \pm 2.18$ & $18.47 \pm 1.67$ & $19.17 \pm 2.8$ \\
\hline
\end{tabular}

The results are expressed as mean \pm SD. No significant difference between different groups of diabetic foot syndrome grading

Table 4. Correlations between serum levels of neopterin [ $\mathrm{nmol} / \mathrm{L}]$ and cardiometabolic risk factors and the inflammatory marker

\begin{tabular}{lcccc}
\hline Independent risk factors & \multicolumn{2}{c}{ T1D } & \multicolumn{2}{c}{ T2D } \\
\cline { 2 - 5 } & Correlation factor $(r)$ & Probability & Correlation factor $(r)$ & Probability \\
\hline Duration of diabetes & -0.118 & 0.337 & +0.042 & 0.733 \\
Duration of DFS & +0.019 & 0.877 & -0.123 & 0.317 \\
Body mass index [kg/m $\left.{ }^{2}\right]$ & +0.082 & 0.506 & +0.170 & 0.165 \\
Waist circumference [cm] & -0.128 & 0.298 & +0.102 & 0.407 \\
Systolic blood pressure [mm Hg] & +0.072 & 0.559 & +0.135 & 0.272 \\
Diastolic blood pressure [mm Hg] & +0.261 & $0.031^{*}$ & +0.042 & 0.733 \\
Fasting serum triglycerides [mg/dL] & +0.219 & 0.072 & +0.248 & $0.041^{*}$ \\
High-density lipoprotein cholesterol [mg/dL] & +0.024 & 0.845 & -0.039 & 0.752 \\
Fasting serum glucose [mg/dL] & -0.038 & 0.758 & +0.167 & 0.173 \\
HbA ${ }_{1 \mathrm{c}}$ (\%) & -0.051 & 0.679 & -0.012 & 0.922 \\
hs-CRP [mg/L] & +0.189 & 0.124 & +0.204 & 0.093 \\
\hline
\end{tabular}

The results are expressed as correlation factor $(r)$ and the corresponding $p$ value

patients $(18.6 \pm 2.1 \mathrm{nmol} / \mathrm{L}$ vs. $12.6 \pm 1.3 \mathrm{nmol} / \mathrm{L})$. The results of this study showed that the alterations in serum levels of neopterin were independently related to cardiometabolic risk factors and that these relations differed depending on the type of diabetes (Tab. 4). In T1D, a significant positive correlation between the serum levels of neopterin and diastolic blood pressure was observed, while in T2D a significant positive correlation was found between fasting serum triglyceride levels and neopterin levels (Tab. 4). Moreover, the changes in the serum neopterin levels were not related to the inflammation. The results of this study showed that serum levels of neopterin were insignificantly correlated with hs-CRP in T1D and T2D (Fig. 1).

\section{Discussion}

The results of this study show that the serum levels of hs-CRP and neopterin are significantly higher in T2D compared with T1D in patients presented with DFS. The cardiometabolic risk factors are associated with DFS, but their levels do not show significant differences between the grades of DFS in T1D and T2D. There were significantly fewer patients with a history of lower extremity amputation in T1D group compared with T2D group, while the frequency of high-grade wound classification (assessed at the time of the study entry), although lower in T1D patient, was only insignificantly different. The results of this study show that the DFS may develop in both T1D and T2D when cardiometa- 


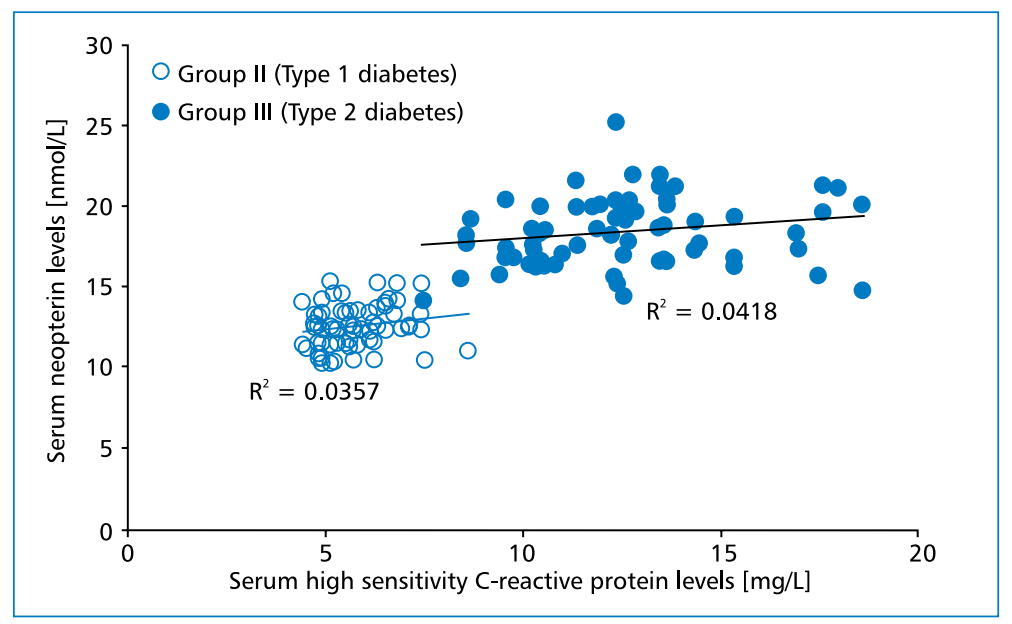

Figure 1. Correlations between serum levels of high-sensitivity C-reactive protein and serum neopterin levels in patients with diabetic foot syndrome

bolic risk factors are present, regardless the fact that the prevalence of the DFS among T1D patients is lower than among those with T2D [14]. Hypertriglyceridemia ( $\geq 150 \mathrm{mg} / \mathrm{dL}$ ) was associated with DFS in both T1D and T2D patients, but the overall mean levels of fasting serum triglycerides in Group II patients were significantly lower than in Group III patients $(168.5 \pm 10.1 \mathrm{mg} / \mathrm{dL}$ vs. $198.6 \pm 17.7 \mathrm{mg} / \mathrm{dL}, \mathrm{p}<0.001)$. These results are in agreement with other studies which reported that dyslipidaemia is a risk factor of DFS $[6,15]$. The levels of fasting serum triglycerides in each group had no significant relation to the Wagner-Meggitt classification of DFS. High blood pressure was observed significantly more frequently in Group III compared with Group II (systolic $148.9 \pm 5.0 \mathrm{~mm} \mathrm{Hg}$ vs. $131.2 \pm 3.5 \mathrm{~mm} \mathrm{Hg}$ and diastolic $90.0 \pm 4.6$ vs. $75.8 \pm 3.4 \mathrm{~mm} \mathrm{Hg}$ ). These findings are compatible with other studies reporting the prevalence of hypertension among diabetic patients with foot ulceration of $57.1 \%$ [16]. Again, blood pressure values were not related to the severity of DFS. All the patients in Group II and Group III were obese and this observation not only supported the hypothesis on the role of adipovascular axis in T2D, but also extended it to T1D [17]. The measurements of diabetic control, i.e. fasting serum glucose and glycated haemoglobin, were worse in T1D compared with T2D patients, but did not show significant variations in respect to the severity of DFS [18]. The results of this study clearly confirm previous reports that significantly higher levels of hs-CRP and neopterin were observed in T2D complicated with foot ulcers [19, 21]. Higher serum levels of neopterin among T2D compared with T1D patients indicates activation of cell-mediated immune response and increase in reactive oxygen species [22,
23]. Previous studies showed that neopterin levels were influenced by proinflammatory and anti-inflammatory cytokines and this explains the insignificant correlation between serum neopterin and hs-CRP levels observed in this study [24]. Two important findings of our study should be highlighted: first one is the lack of relationship between serum neopterin levels and inflammation; and the second finding is the observation that serum neopterin correlation with metabolic risk factors depends on the type of diabetes. A significant correlation between blood pressure and serum levels of neopterin in T1D patient is a new finding and confirms previous studies that showed increased serum neopterin levels in preeclampsia [25]. The other significant positive correlation of neopterin was with the serum levels of triglyceride in T2D, which confirmed the results of previous studies [26]. One of the limitations of our study is small sample size, which precluded statistical analysis of serum neopterin levels and their correlations with cardiometabolic risk factors in respect to the grading and severity of cardiometabolic risk factors.

There is no doubt that dyslipidaemia, obesity and high blood pressure are contributing factors that accelerate the development of micro- and macrovascular complications in diabetes, including DFS. This study accepted the hypothesis that cardiometabolic risk factors play a role in the development of diabetic complications.

\section{Conclusions}

We conclude that cardiometabolic risk factors are associated with DFS in T1D and T2D and that their levels are not related to DFS severity assessed according to the Wagner-Meggitt classification. Serum levels of neo- 
pterin are significantly increased in DFS and are higher inT2D compared with T1D patients. Determination of serum neopterin level can serve as a cardiometabolic risk factor rather than an inflammatory marker and the type of diabetes should be considered in the interpretation of the changes in serum neopterin levels.

\section{Acknowledgement}

The authors expressed a great gratitude to the presidency of the Hawler Medical University and the members of the Diabetic Centre in Erbil for their unlimited help in performing this study.

\section{Conflict of interest}

There are no conflicts of interest.

\section{REFERENCES}

1. Jeffcoate WJ, Macfarlane RM, Fletcher EM. The description and classification of diabetic foot lesions. Diabet Med. 1993; 10(7): 676-679, doi: 10.1111/j.1464-5491.1993.tb00144.x, indexed in Pubmed: 8403832.

2. Richard JL, Schuldiner S. [Epidemiology of diabetic foot problems]. Rev Med Interne. 2008; 29 Suppl 2: S222-S230, doi: 10.1016/ /S0248-8663(08)73949-3, indexed in Pubmed: 18822247.

3. Nather A, Bee CS, Huak CY, et al. Epidemiology of diabetic foot problems and predictive factors for limb loss. J Diabetes Complications. 2008; 22(2): 77-82, doi: 10.1016/j.jdiacomp.2007.04.004, indexed in Pubmed: 18280436.

4. AlAyed MY, Younes N, Al-Smady M, et al. Prevalence of Foot Ulcers, Foot at Risk and Associated Risk Factors Among Jordanian Diabetics. Curr Diabetes Rev. 2017; 13(2): 182-191, doi: 10.2174/ /1573399812666151210143140, indexed in Pubmed: 26652612.

5. Lauterbach S, Kostev K, Kohlmann T. Prevalence of diabetic foot syndrome and its risk factors in the UK. J Wound Care. 2010; 19(8): 333-337, doi: 10.12968/jowc.2010.19.8.77711, indexed in Pubmed: 20852505.

6. Zubair M, Malik A, Ahmad J. Plasma adiponectin, IL-6, hsCRP, and TNF- $\alpha$ levels in subject with diabetic foot and their correlation with clinical variables in a North Indian tertiary care hospital. Indian J Endocrinol Metab. 2012; 16(5): 769-776, doi: 10.4103/22308210.100672, indexed in Pubmed: 23087862

7. Noor S, Borse AG, Ozair M, et al. Inflammatory markers as risk factors for infection with multidrug-resistant microbes in diabetic foot subjects. Foot (Edinb). 2017; 32: 44-48, doi: 10.1016/j. foot.2017.05.001, indexed in Pubmed: 28802182

8. Schoedon G, Troppmair J, Fontana A, et al. Biosynthesis and metabolism of pterins in peripheral blood mononuclear cells and leukemia lines of man and mouse. Eur J Biochem. 1987; 166(2): 303-310, doi: 10.1111/j.1432-1033.1987.tb13515.x, indexed in Pubmed: 3301338.

9. Grossmann V, Schmitt VH, Zeller T, et al. Profile of the Immune and Inflammatory Response in Individuals With Prediabetes and Type 2 Diabetes. Diabetes Care. 2015; 38(7): 1356-1364, doi: 10.2337/dc14-3008, indexed in Pubmed: 25877811.

10. Rao VS, Nagaraj RK, Hebbagodi S, et al. Association of inflammatory and oxidative stress markers with metabolic syndrome in asian indians in India. Cardiol Res Pract. 2010; 2011: 295976, doi: 10.4061/2011/295976, indexed in Pubmed: 21234321.
11. Karaca A, Omma T, Dura Deveci C, et al. Neopterin and hsCRP are not correlated in gestational diabetes mellitus. Gynecol Endocrinol. 2016; 32(12): 977-981, doi: 10.1080/09513590.2016.1193850, indexed in Pubmed: 27276070.

12. Vengen IT, Dale AC, Wiseth R, et al. Neopterin predicts the risk for fatal ischemic heart disease in type 2 diabetes mellitus: long-term follow-up of the HUNT 1 study. Atherosclerosis. 2009; 207(1): 239-244, doi: 10.1016/j.atherosclerosis.2009.04.003, indexed in Pubmed: 19409566.

13. James WB. Classification of foot lesion in diabetic patients. In: Bowker JH, Pfeifer MA. ed. Levin and O'Neal's The Diabetic Foot. 7th edition. Mosby, Philadelphia 2008: 221-226.

14. Marno T, Yifter $H$, Lemessa T. Risk factors assessment of diabetic foot ulcer using the sixty seconds screening tools: A hospital based cross-sectional study at Tikur Anbessa specialized hospital. Ethiop Med. 2015(Suppl 2): 45-49, indexed in Pubmed: 26591282.

15. Chatzistergos PE, Naemi R, Sundar $L$, et al. The relationship between the mechanical properties of heel-pad and common clinical measures associated with foot ulcers in patients with diabetes. J Diabetes Complications. 2014; 28(4): 488-493, doi: 10.1016/j. jdiacomp.2014.03.011, indexed in Pubmed: 24795257.

16. Wu L, Hou Q, Zhou Q, et al. Prevalence of risk factors for diabetic foot complications in a Chinese tertiary hospital. Int J Clin Exp Med. 2015; 8(3): 3785-3792, indexed in Pubmed: 26064275.

17. Tuttolomondo A, Maida C, Pinto A. Diabetic foot syndrome: Immune-inflammatory features as possible cardiovascular markers in diabetes. World J Orthop. 2015; 6(1): 62-76, doi: 10.5312/wjo. v6.i1.62, indexed in Pubmed: 25621212.

18. Al-Rubeaan K, Al Derwish M, Ouizi S, et al. Diabetic foot complications and their risk factors from a large retrospective cohort study. PLoS One. 2015; 10(5): e0124446, doi: 10.1371/journal. pone.0124446, indexed in Pubmed: 25946144.

19. Arık HO, Yalcin AD, Gumuslu $S$, et al. Association of circulating sTRAIL and high-sensitivity CRP with type 2 diabetic nephropathy and foot ulcers. Med Sci Monit. 2013; 19: 712-715, doi: 10.12659/MSM.889514, indexed in Pubmed: 23986130.

20. Wang Ph, Yu Dm, Chu Yj, et al. [Research on the clinical features and effective factors of 249 diabetic patients with deep foot infection]. Zhonghua Yi Xue Za Zhi. 2007; 87(26): 1828-1831, indexed in Pubmed: 17922992.

21. Eisenhut M. Neopterin in Diagnosis and Monitoring of Infectious Diseases. J Biomark. 2013; 2013: 196432, doi: 10.1155/2013/196432, indexed in Pubmed: 26317013.

22. Pingle SK, Tumane RG, Jawade AA. Neopterin: Biomarker of cellmediated immunity and potent usage as biomarker in silicosis and other occupational diseases. Indian J Occup Environ Med. 2008; 12(3): 107-111, doi: 10.4103/0019-5278.44690, indexed in Pubmed: 20040967.

23. Zis P, Strydom A, Buckley D, et al. Cognitive ability in Down syndrome and its relationship to urinary neopterin, a marker of activated cellular immunity. Neurosci Lett. 2017; 636: 254-257, doi: 10.1016/j.neulet.2016.11.023, indexed in Pubmed: 27851899.

24. Kolb H, Lückemeyer K, Heise T, et al. DIATOR Study Group. The systemic immune network in recent onset type 1 diabetes: central role of interleukin-1 receptor antagonist (DIATOR Trial). PLoS One. 2013; 8(8): e72440, doi: 10.1371/journal.pone.0072440, indexed in Pubmed: 23991111.

25. Amash A, Holcberg G, Sapir O, et al. Placental secretion of interleukin-1 and interleukin-1 receptor antagonist in preeclampsia: effect of magnesium sulfate. J Interferon Cytokine Res. 2012; 32(9): 432-441, doi: 10.1089/jir.2012.0013, indexed in Pubmed: 22909148.

26. Gottsäter A, Forsblad J, Mätzsch T, et al. Interleukin-1 receptor antagonist is detectable in human carotid artery plaques and is related to triglyceride levels and Chlamydia pneumoniae $\lg \mathrm{A}$ antibodies. J Intern Med. 2002; 251(1): 61-68, doi: 10.1046/j.13652796.2002.00926.x, indexed in Pubmed: 11851866. 